\title{
Advanced breakup-nucleon enhancement of deuteron-induced reaction cross sections
}

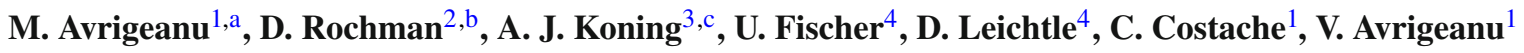 \\ ${ }^{1}$ Horia Hulubei National Institute for Physics and Nuclear Engineering, P.O. Box MG-6, 077125 Bucharest-Magurele, Romania \\ ${ }^{2}$ Laboratory for Reactor Physics and Thermal-Hydraulics, Paul Scherrer Institut, 5232 Villigen, Switzerland \\ ${ }^{3}$ International Atomic Energy Agency, Vienna International Centre, PO Box 100, 1400 Vienna, Austria \\ ${ }^{4}$ Karlsruhe Institute of Technology (KIT), Hermann-von-Helmholtz-Platz, 1, 76344 Eggenstein-Leopoldshafen, Germany
}

Received: 29 October 2021 / Accepted: 27 December 2021 / Published online: 10 January 2022

(C) The Author(s) 2022

Communicated by D. Lacroix

\begin{abstract}
Following the EUROfusion PPPT-programme action for an advanced modeling approach of deuteroninduced reaction cross sections, as well as specific data evaluations in addition of the TENDL files, an assessment of the details and corresponding outcome for the latter option of TALYS for the breakup model has been carried out. The breakup enhancement obtained in the meantime within computer code TALYS, by using the evaluated nucleon-induced reaction data of TENDL-2019, is particularly concerned. Discussion of the corresponding results, for deuteron-induced reactions on ${ }^{58} \mathrm{Ni},{ }^{96} \mathrm{Zr}$, and ${ }^{231} \mathrm{~Pa}$ target nuclei up to $200 \mathrm{MeV}$ incident energy, includes limitations still existing with reference to the direct-reaction account.
\end{abstract}

\section{Introduction}

The design analyses of the high intensity accelerator-based neutron source International Fusion Materials Irradiation Facility (IFMIF) [1], for ITER fusion device [2] and European DEMO fusion reactor [3] material qualification, require also high-quality cross-section data of deuteron induced reactions. Related activities are currently conducted within the Power Plant Physics and Technology (PPPT) programme of the EUROfusion Consortium [4], following a previous partnership agreement of Fusion for Energy (F4E) [5] with the Consortium on Nuclear Data Development and Analysis [6]. A sub-library of the latest version 3.2 [7] of Fusion Evaluated Nuclear Data Library (FENDL) [8], developed at IAEA/NDS, is going to include the most recent correspond-

\footnotetext{
a e-mail: marilena.avrigeanu@ @ipne.ro (corresponding author)

b e-mail: dimitri-alexandre.rochman@psi.ch

c e-mail: a.koning@iaea.org
}

ing results, while there is a world-wide use of the deuteron sub-library of the current TENDL-2019 evaluated nuclear data library [9] based on the output of the TALYS nuclear model code system [10] for direct use in both basic research and applications.

The TENDL deuteron data library was in fact the current reference used in the PPPT project of the Early Neutron Source (ENS), the need of its further improvement being recently pointed out [11]. The dedicated effort already allocated in the PPPT programme including also contributions from all involved deuteron reaction mechanisms [12] has been concerned in this respect. It followed specific data evaluations which have complemented and updated the TENDL files which are based to a large extend on automated nuclear model calculations with the TALYS code using default models and parameters [13].

Actually, the mechanisms of deuteron-induced reactions are more complex than those of nucleon-induced ones due to the deuteron weakly bound nature. Thus, owing to its binding energy $B_{d}=2.224 \mathrm{MeV}$, the deuteron easily breaks up in the Coulomb and nuclear fields of a target nucleus. Then, this already intricate process involves also a variety of reactions initiated by the neutron and proton that follow the deuteron breakup (BU) $[14,15]$, i.e. the elastic breakup (EB) in which the target nucleus remains in its ground state and none of the deuteron constituents interacts inelastically with it, and the inelastic breakup or breakup fusion (BF) [16], where one of these deuteron constituents interacts nonelastically with the target nucleus throughout breakup states in the continuum. Therefore, the usual low-energy reaction mechanisms of direct reaction (DR), pre-equilibrium emission (PE) and compound-nucleus $(\mathrm{CN})$ de-excitation should be considered not only for the incident deuterons on a target nucleus but 
also for reactions induced by breakup-nucleons on the same nucleus.

On the other hand, these specific issues made possible projects of highly efficient fast-neutron sources by using deuterons accelerated on light nuclei (isotopes of $\mathrm{Li}, \mathrm{Be}$, C, etc), including IFMIF, NFS [17], and SARAF [18]. At the same time, deuteron activation of various structural materials $(\mathrm{Al}, \mathrm{Fe}, \mathrm{Ni}$, etc.) within deuteron-accelerator components needs also a suitable assessment, despite of a scarce experimental data basis, too. Therefore, accurate and comprehensive deuteron nuclear data libraries over wide ranges of incident energies and target mass from light elements to structural materials, are mandatory for the engineering design of a deuteron-accelerator neutron source facility as IFMIF.

Nevertheless, TENDL-2017 deuteron sub-library improvement is based on the use in TALYS of the latest phenomenological BU model by Kalbach [19], following the analysis of an extensive database of double-differential cross sections for the breakup of deuterons, ${ }^{3} \mathrm{He}$ ions, and $\alpha$ particles. Thus, global predictions for the absorptive breakup cross section have been involved, at once with Kalbach's pick-up and stripping models [20] for all combinations of projectiles and ejectiles. However, while it was decided to adopt TENDL-2017 for FENDL-3.2 charged-particle induced activation files, data from the incident chargedparticle medical isotope cross section data [21], if available, are taken in preference to TENDL-2017 [22]. This decision followed the recommendation of full parametrization of the available data, by a least-squares fit with Padé approximations of variable order, for deuteron monitor reactions and therapeutic radionuclides-production cross sections [23,24].

In fact, deficiencies in describing the elastic and especially the inelastic BU that dominates the deuteron reaction cross section at beam energies comparable with the Coulomb barrier motivated this recommendation [24], however with no predictive power. Earlier, a simple phenomenological description of the available stripping $(d, p)$ experimental data was also proposed and included in the codes ALICE-D and EMPIRE-D [25]. It includes six parameters obtained by analysis of the excitation functions of four target nuclei, from ${ }^{59} \mathrm{Co}$ to ${ }^{197} \mathrm{Au}$, and estimated accuracy of about 25-30\%. However, its further use has not been reported yet while another energy-dependent enhancement factor $K$ has most recently been mentioned without either definite values or model analysis [26,27].

Following the above-mentioned PPPT-programme action for an advanced modeling approach and specific data evaluations in addition of the TENDL files [11,12], an assessment of the details and corresponding outcome related to a latter option of TALYS for the breakup model makes the object of this work. An overview of the model approaches related to deuteron nuclear data for the last decade and a half, including the activities conducted recently in the PPPT programme as a first step of the flow chart linking modern theoretical nuclear physics to large-scale nuclear energy systems [28], is given in Sect. 2 of this paper. The consequent assumptions and add-ons proved useful in the meantime within TALYS, concerning particularly the breakup enhancement of the deuteron-induced reaction cross sections, are subject of Sect. 3. The corresponding results and their discussion including limitations still existing with reference to the direct-reaction account, are shown in Sect. 4, as well as conclusions in Sect. 5.

\section{Applied theory overview}

\subsection{Deuterons on light nuclei}

In order to improve the calculation of the $d$ - $L i$ neutron source term of IFMIF, an analysis of the elastic-scattering angular distributions of deuterons on ${ }^{6,7} \mathrm{Li}$, for energies from 3 up to $50 \mathrm{MeV}$, using a phenomenological optical model potential (OMP) [29] was involved within an updated $d+{ }^{6,7} \mathrm{Li}$ data evaluation [30,31]. The OMP analysis was completed by the coupled reaction channels (CRC) method within the code FRESCO [32] for consideration of the elastic transfer additional mechanism [33]. The reliability of the deduced particular and average OMP parameters was proved at variance with the extrapolation of the deuteron global potentials over a wider range of mass and energy domains. The key output of this analysis has been the deuteron total-reaction cross sections that are involved subsequently within all related data evaluation. A clear improvement of the prediction accuracy for the IFMIF neutron source term simulation was proved through benchmark calculations against experimental thick lithium target neutron yields [30,31].

Also in connection with the IFMIF project, Ye et al. analyzed in Ref. [34] the deuteron elastic scattering from ${ }^{6,7} \mathrm{Li}$ in the energy range from 10 to $50 \mathrm{MeV}$ by the continuum discretized coupled channels (CDCC) method ([35] and Refs. therein). They paid thus due attention to the deuteron breakup by a quantum-mechanical approach, taking into account that intermediate-energies semiclassical models were used previously [30] at a relatively low energy and neglecting the EB contribution. The deuteron breakup process is indeed involved within CDCC method by explicitly using a phenomenological three-body Hamiltonian in which the nucleon-nucleus interaction is represented by the OMP at half the deuteron incident energy and an effective nucleonnucleon potential is used for the $p-n$ interaction. A good agreement was obtained at forward angles to the same extent as the optical model calculation with the phenomenological deuteron OMP [29], while a reduction of the elasticscattering angular distributions at large angles was found due to the BU account. 
The analysis of the deuteron EB in terms of the CDCC method was then completed in Ref. [36] by that of the nonelastic breakup (with the notation STR for nucleonstripping in the continuum, at that time) using the semiclassical approach of the Glauber model [37] to account for angular distribution and double differential cross sections (DDX) of $(d, x n)$ and $(d, x p)$ reactions on ${ }^{7} \mathrm{Li}$ at deuteron energies of 40 and $100 \mathrm{MeV}$, respectively. This investigation of the Glauber model applicability at incident energies below $40 \mathrm{MeV} /$ nucleon proved successful at forward angles, showing also that the nonelastic breakup component is more important than EB in deuteron reactions on ${ }^{7} \mathrm{Li}$. Shapes and magnitudes of the experimental $(d, x p)$ spectra at $100 \mathrm{MeV}$ have been reproduced at forward angles $\left(<20^{\circ}\right)$ also for the target nuclei ${ }^{9} \mathrm{Be}$ and ${ }^{12} \mathrm{C}$ [38]. Results of a CDCC extension [39] for the DDX data of $(d, x n)$ reaction on ${ }^{7} \mathrm{Li}$ at $40 \mathrm{MeV}$ seems to be only slightly better than the Glauber-model ones.

Moreover, a modification of the Glauber model that was found necessary at low energies, to take into account the much stronger effects of the nuclear and Coulomb potentials than those at high energies, corresponding to a projectile curved trajectory [40], improved the total-reaction and EB cross sections for six target nuclei, from ${ }^{7} \mathrm{Li}$ to ${ }^{208} \mathrm{~Pb}$, at energies up to $100 \mathrm{MeV} /$ nucleon. However, despite of remarkable results, the theoretical foundation of this semi-classical model was considered still questionable in the low energy region where the wavelength of the projectile becomes large and the classical trajectory approximation becomes worse.

In addition to the EB and nonelastic breakup modeled by CDCC method and Glauber model [39], respectively, the account of the stripping reactions to bound states of the residual nuclei through zero-range distorted wave Born approximation (DWBA) was considered within angular-distribution analysis for the $(d, p)$ reaction on ${ }^{12} \mathrm{C}$ as well as heavier nuclei ${ }^{27} \mathrm{Al},{ }^{40} \mathrm{Ca}$, and ${ }^{58} \mathrm{Ni}$, at deuteron energies from 7 to $56 \mathrm{MeV}$ [41]. This BU + DR analysis was completed by $\mathrm{PE}+\mathrm{CN}$ contributions provided by the exciton and HauserFeshbach statistical models. DDX data integrated over the outgoing energy, for incident energies from 56 to $100 \mathrm{MeV}$, were similarly analyzed as well as angular-distributions for the ${ }^{12} \mathrm{C}(d, n){ }^{13} \mathrm{~N}$ reaction at incident energies from 7 to 18 $\mathrm{MeV}$ [42]. Of particular interest has been a similar, even if less clear, energy dependence of the spectroscopic factors extracted from DWBA analyses for the $(d, p)$ reaction on all the four target nuclei, under the assumption that the incidentenergy dependence is same for all final states. An empirical expression of this energy dependence has been obtained, with various scaling factors for each target nucleus and residual state, while finally was considered that it might not be possible to deduce such a simple empirical formula.

The above-mentioned CDCC-Glauber-DWBA analysis completed by $\mathrm{PE}+\mathrm{CN}$ contributions within deuteroninduced reaction analysis code system DEURACS, for
DDX data of $(d, x n)$ reactions on ${ }^{9} \mathrm{Be}$ and ${ }^{12} \mathrm{C}$, at incident energies up to $50 \mathrm{MeV}$, took into account in Ref. [43] also the $\mathrm{PE}+\mathrm{CN}$ decay following the absorption by the target nucleus of either a BU-neutron or a BU-proton in addition to a deuteron itself. An incoherent summation of the EB and nonelastic-breakup components was thus explicitly enlarged by three statistical-decay components, corresponding to the three distinct compound nuclei and weighted by nonelasticbreakup formation fractions $R_{d}, R_{n}$, and $R_{p}$ calculated with the Glauber model [39]. The additional $(n, x n)$ and $(p, x n)$ components were calculated for nucleon-incident energies at half the deuteron incident energy for saving computational time, while the DDX data were well reproduced quantitatively over a wide range of neutron emission energy except the energies below a few $\mathrm{MeV}$.

Recently, energy-dependent formation factors $R_{n}\left(E_{n}\right)$ and $R_{p}\left(E_{p}\right)$ have been involved in Ref. [44] within the same DEURACS framework, as given by Glauber model [39] for the nonelastic-breakup (NBU, replacing the authors' former notation STR). The related analysis of DDX data for $(d, x n)$ reactions on ${ }^{9} \mathrm{Be}$ and ${ }^{12} \mathrm{C}$, at incident energy of $102 \mathrm{MeV}$, has proved the applicability of DEURACS to the $(d, x n)$ reactions on light nuclei at incident energies around $100 \mathrm{MeV}$, as well as the essential NBU role of for accurate predictions. On the other hand, it has been pointed out that the related NBU model may not be valid for low incident energy close to the Coulomb barrier, so that it needs to be additionally tested for such data. Nevertheless, a deuteron nuclear data library up to $200 \mathrm{MeV}$ for ${ }^{7} \mathrm{Li},{ }^{9} \mathrm{Be}$, and ${ }^{12,13} \mathrm{C}$ has been developed on this basis, with particular attention to neutron production in Ref. [45]. The above-mentioned underestimation below a few $\mathrm{MeV}$, which was attributed to an absent particle-decay from discrete levels in residual light nuclei $[43,44]$, has also been amended.

\subsection{Deuterons on increasing-mass nuclei}

\subsubsection{Breakup-parametrization based model analysis}

Former analysis of deuteron-induced activation data for IFMIF calculations showed that the deuterons are much more important than the neutrons (about a factor of 70) in activating elements other than lithium, so that refinement of the model calculations and further experimental measurements are needed if the deuteron libraries are to approach the standard of the established neutron ones [46], for a suitable input into IFMIF engineering validation and engineering design activities (EVEDA) phase.

Along this line, an increased attention was firstly provided to BU effects as well as the simultaneous analysis of the deuteron elastic-scattering and induced activation cross sections as for ${ }^{27} \mathrm{Al}$ in Ref. [47]. Thus, a parametrization of both $\mathbf{E B}$ cross section $\sigma_{E B}$ and total $\mathbf{B U}$ proton-emission 
cross section $\sigma_{B U}^{p}=\sigma_{E B}+\sigma_{B F}^{p}$ was obtained by analysis of experimental systematics of deuteron-induced reactions on target nuclei from ${ }^{27} \mathrm{Al}$ to ${ }^{232} \mathrm{Th}[14,16,48-50]$. Equal BF nucleon-emission cross sections $\sigma_{B F}^{n}$ and $\sigma_{B F}^{p}$ were assumed [16], so that the total BU cross section is $\sigma_{B U}=\sigma_{E B}+2 \sigma_{B F}^{n / p}$. It should be underlined that the experimental data $[14,16,48-$ 50] were obtained through a distinct analysis of the BU, DR, $\mathrm{PE}$, and $\mathrm{CN}$ reaction mechanisms.

Consequently $\sigma_{B U}$ was subtracted from deuteron totalreaction cross section $\sigma_{R}$ that should be shared among different $\mathrm{DR}+\mathrm{PE}+\mathrm{CN}$ outgoing channels, while the BF component brings additional contributions to different $(d, x)$ reaction channels through $(n, x)$ and $(p, x)$ reactions on the same target nucleus ${ }^{27} \mathrm{Al}$. This breakup enhancement of various $(d, x)$ reaction cross sections by breakup-neutrons through $(n, x)$ reactions was taken as the product between $\sigma_{B F}^{n}$ and the ratio $\sigma_{(n, x)} / \sigma_{n o n}$, where $\sigma_{n o n}$ is the neutron non-elastic cross section. The similar effect of the $(p, x)$ reactions was given by the equivalent $\sigma_{B F}^{p}$ multiplied by the ratio $\sigma_{(p, x)} / \sigma_{R}$, where $\sigma_{R}$ is the proton total-reaction cross section. The two ratios were expressed as a function of the deuteron incident energy using the Kalbach [51] formula for the center-of-mass system centroid energy of the deuteron-breakup peak energies of the emitted constituents.

At the same time there have been used: (i) a semimicroscopic OMP based on a double-folding model (DFM) real part, and phenomenological imaginary and spin-orbit terms together with dispersive corrections to the DFM results, and (ii) a related phenomenological OP real part replacing the DFM one, both of them being proved by analysis of deuteron elastic-scattering data at energies from 5 to $59 \mathrm{MeV}$, and $\sigma_{R}$ at $\sim 13$ and $25 \mathrm{MeV}$, as well as (iii) the DR contributions obtained with code FRESCO [32] and DWBA spectroscopic factors from data analysis of $(d, p)$ angular distributions for 35 final states of the residual nucleus ${ }^{28} \mathrm{Al}$ up to $5.135 \mathrm{MeV}$, at incident energies of 6,12 , and $23 \mathrm{MeV}$, and also $(d, n)$ angular distributions for 18 final states of ${ }^{28} \mathrm{Si}$ up to $10.38 \mathrm{MeV}$, at incident energy of $6 \mathrm{MeV}$ [47], and (iv) usual PE + CN contributions of the code STAPRE-H [52]. This simultaneous analysis of the deuteron elastic-scattering and induced activation cross sections including new data measured at incident energies from 3 up to $20 \mathrm{MeV}$ was finally providing suitable account of all available excitation functions, up to the incident energy of $60 \mathrm{MeV}$, not only for the $(d, p)$ but $(d, \alpha)$, $(d, 2 n),(d, 2 p)$, and $(d, p \alpha)$ reactions, too [53].

A further point concerned an apparent decrease of the fraction $f_{E B}=\sigma_{E B} / \sigma_{R}$ at energies beyond the range $E<30$ $\mathrm{MeV}$ of the EB data [14,16], unlike the trend of both the fraction $f_{B U}^{p}=\sigma_{B U}^{p} / \sigma_{R}$ and $\sigma_{R}$. Consequently, the correctness of this EB extended parametrization has been checked in Ref. [54] through the comparison of its predictions and results of the $\mathbf{C D C C}$ method for the ${ }^{63} \mathrm{Cu}$ and ${ }^{93} \mathrm{Nb}$ tar- get nuclei, at incident energies up to $50 \mathrm{MeV}$. Following a CDCC suitable account of experimental elastic-scattering angular distributions of deuterons on ${ }^{63} \mathrm{Cu}$ and ${ }^{93} \mathrm{Nb}$ at energies between 12 and $52 \mathrm{MeV}$, a good agreement was found between the EB data [14] and the corresponding CDCC results as well as empirical parametrization [47] at the energies of the available data. However, $f_{E B}$ becomes significantly lower at higher energies. Therefore, extrapolation of $f_{E B}$ empirical parametrization beyond the energies of the corresponding data have to be considered with caution, at the same time with the challenging CDCC calculations for each target/energy of interest. In the meantime a normalization of the EB fraction for the energies beyond the maximum of the former parametrization [47] has been adopted in Ref. [55] by taking unchanged the ratio of the EB and BU fractions at the incident energies above the $f_{E B}$ maximum.

However, the former breakup-enhancement broad approximation [47] using only the centroid energies of the breakup nucleons [51], was at variance with quite large widths of the assumed Gaussian distribution of the corresponding deuteron-breakup nucleon energies, for a given deuteron incident energy. Therefore it has been replaced by a convolution of the each one of energy-dependent ratio $\sigma_{(n, x)} / \sigma_{n o n}$ or $\sigma_{(p, x)} / \sigma_{R}$, and the related Gaussian line shape. The corresponding distributions and convolution results, at various deuteron energies, shown for deuterons incident on $\mathrm{Cu}$ [56], ${ }^{231} \mathrm{~Pa}$ [57], and ${ }^{93} \mathrm{Nb}$ [58] proved the need for this improvement, with the detailed formula given elsewhere $[59,60]$. Additionally, the key role of BU and DR processes in deuteron-induced reactions has been pointed out in Ref. [12] by the differences between larger $\mathrm{CN}$ contributions obtained from measured neutron angular distributions, with no BU consideration, and $\mathrm{BU}+\mathrm{DR}+\mathrm{PE}+\mathrm{CN}$ analyses.

Actually, the same approach beyond elastic-scattering analysis for OMP validation, using BU parametrization $[47,55]$ for the related deuteron $\sigma_{R}$ decrease as well as breakup-enhancement of various $(d, x)$ reaction cross sections, DWBA spectroscopic factors from data analysis with FRESCO of available particle-emission angular distributions, and PE + CN framework of either STAPRE-H or then TALYS codes has been consistently involved within suitable account of all available excitation-function data of deuterons up to $60 \mathrm{MeV}$ on ${ }^{27} \mathrm{Al}[53],{ }^{63,65, \text { nat }} \mathrm{Cu}[18,56]$, ${ }^{93} \mathrm{Nb}$ [58], ${ }^{54,56,57,58, \text { nat }} \mathrm{Fe}[60],{ }^{58,60,61,62,64, \text { nat }} \mathrm{Ni}$ [61], 50,52,53,54,nat $\mathrm{Cr}[62],{ }^{51, \text { nat }} \mathrm{V}$ [63], ${ }^{59} \mathrm{Co}[63],{ }^{55} \mathrm{Mn}$ [64], and 90,91,92,94,96, nat $\mathrm{Zr}$ [65]. Due consideration of all EB, BF, DR, $\mathrm{PE}$, and $\mathrm{CN}$ reaction mechanisms $[60,61]$ is indeed found crucial in Ref. [66] for a consistent analysis of the deuteronreaction measured data and even high production of protonrich nuclei [67], while insufficient treatment and separation between different reaction mechanisms such as DR and BU components [68] may be related to deviations between measurements and advanced surrogate reaction studies [69]. On 
the other hand, the concerned choice for the BU fractions has thus been validated.

\subsubsection{Breakup-semi-classical account}

On the other hand, at once with the work on deuteron-induced reactions on $\mathrm{Li}, \mathrm{Be}$, and $\mathrm{C}$ isotopes, the above-mentioned CDCC results [39] reproduced also experimental elasticscattering angular distribution of deuterons on ${ }^{58} \mathrm{Ni}$ at 200 $\mathrm{MeV} /$ nucleon, while the Glauber model was found good for light targets, as expected, but not for EB and nonelastic breakup for heavy targets. At the same time, the particular attention paid to the deuteron BU over a wide mass range of target nuclei from ${ }^{9} \mathrm{Be}$ to ${ }^{238} \mathrm{U}$ [38] has proved shapes and magnitudes of the experimental $(d, x p)$ spectra at $100 \mathrm{MeV}$ which are reproduced at forward angles $\left(<20^{\circ}\right)$ for target nuclei less than ${ }^{58} \mathrm{Ni}$ but underestimated the specific bump around half the incident energy as the target mass increased. It was thus concluded that more quantummechanical approaches beyond the Glauber model would be necessary to account for $(d, x p)$ reactions for heavier-mass nuclei at relatively low incident energies below $100 \mathrm{MeV}$.

Similar results have been obtained for elastic-scattering angular distribution of deuterons on ${ }^{27} \mathrm{Al}$ and ${ }^{58} \mathrm{Ni}$ at incident energies between $\sim 11-79 \mathrm{MeV}$, as well as for corresponding DDX data of $(d, x p)$ reactions at 56 and $100 \mathrm{MeV}$ [70]. An additional note may concern in this case the inclusion of $\mathrm{PE}+\mathrm{CN}$ contributions within the exciton and HauserFeshbach models, corresponding to the absorption by the target nucleus of either a BU-neutron or a BU-proton in addition to a deuteron itself. The related NBU formation fractions $R_{n}, R_{p}$, and $R_{d}$ were calculated with the Glauber model $[39,40]$ and the assumption that the incident energies of proton and neutron are half the deuteron incident energy. Actually, the additional two statistical-decay components correspond to the former breakup-enhancement approach [53] that involved BF cross sections [47] and BU-nucleon centroid energies of Kalbach [51].

The above-mentioned CDCC-Glauber-DWBA analysis, including the $\mathrm{PE}+\mathrm{CN}$ components as above, of the $(d, p)$ reaction also on ${ }^{27} \mathrm{Al},{ }^{40} \mathrm{Ca}$, and ${ }^{58} \mathrm{Ni}[41]$ has reproduced also the experimental angular-distributions of the $(d, p)$ reaction on ${ }^{27} \mathrm{Al}$ at 6,12 , and $23 \mathrm{MeV}[71]$ as well as ${ }^{27} \mathrm{Al}(d, p)^{28} \mathrm{Al}$ excitation function from the threshold to $20 \mathrm{MeV}$. More recent DEURACS analysis of $(d, x p)$ reactions on ${ }^{27} \mathrm{Al}$ at incident energies of 56 and $100 \mathrm{MeV}$ [72] has reproduced fairly well the DDX data including both the NBU broad peak at half the incident energy, and the peak structure due to the stripping DR to bound states. The same approach has been proved successful for the account of ${ }^{45} \mathrm{Sc}(d, p)^{46} \mathrm{Sc}$ excitation function for incident energies up to $50 \mathrm{MeV}$.

At the same time, DDX data of $(d, x d)$ reactions on ${ }^{27} \mathrm{Al}$ and ${ }^{58} \mathrm{Ni}$ at incident energies of 80 and $100 \mathrm{MeV}$, respectively [44], have been better reproduced than the original PE + CN models due particularly to inclusion of the Kalbach's semi-empirical inelastic scattering model [73]. Energy-dependent formation factors $R_{n}\left(E_{n}\right)$ and $R_{p}\left(E_{p}\right)$, given by the Glauber model $[39,40]$ for the NBU processes, have been also involved in a way rather similar to the above-mentioned BU enhancement based on the convolution of breakup-nucleons energy distributions and the related nucleon-induced reaction excitation functions [56-60].

A conclusive assessment is that, 'taking into account that the CDCC method and the Glauber model have been widely used in analyses at incident energies above 200 MeV/nucleon, it is expected that the framework of DEURACS for the breakup processes is applicable to the incident energy range of 100-200 MeV/nucleon' [74]. This approach has thus been considered in Refs. $[74,75]$ as being complementary to the method using the deuteron-breakup empirical formulas based on data up to $80 \mathrm{MeV}$ [47] and proved successful for reactions at relatively low incident energies below $60 \mathrm{MeV}$ $[53,56-58,60,62,63]$.

\section{Breakup-parametrization framework}

\subsection{Empirical parametrization}

Present analysis of the deuteron breakup mechanism is based on parametrization $[12,47,54,55]$ of both the total breakup $(\mathrm{EB}+\mathrm{BF})$ and $\mathrm{EB}$ data, under the assumption that the inelastic-breakup cross section for neutron emission $\sigma_{B F}^{n}$ is the same as that for the proton emission $\sigma_{B F}^{p}$ [16]. Thus, the total breakup cross sections $\sigma_{B U}$ is given by the sum $\sigma_{E B}$ $+2 \sigma_{B F}^{n / p}$. The parametrization has concerned the total BU nucleon-emission and EB fractions, i.e. $f_{B U}^{n / p}=\sigma_{B U}^{n / p} / \sigma_{R}$ and $f_{E B}=\sigma_{E B} / \sigma_{R}$, respectively, where $\sigma_{R}$ is the deuteron totalreaction cross section. The dependence of these fractions on the deuteron incident energy $E$ and the target-nucleus atomic $Z$ and mass $A$ numbers was obtained $[47,55]$ through analysis of the experimental systematics of deuteron-induced reactions on target nuclei from ${ }^{27} \mathrm{Al}$ to ${ }^{232} \mathrm{Th}$ and incident energies up to $80 \mathrm{MeV}$ for the former $[14,48-50]$,

$$
\begin{aligned}
f_{B U}^{n / p}= & 0.087-0.0066 Z+0.00163 Z A^{1 / 3} \\
& +0.0017 A^{1 / 3} E-0.000002 Z E^{2},
\end{aligned}
$$

but within a more restricted energy range up to $30 \mathrm{MeV}$ [14, 16,48-50] for the latter:

$$
\begin{array}{r}
f_{E B}=0.031-0.0028 Z+0.00051 Z A^{1 / 3} \\
+0.0005 A^{1 / 3} E-0.000001 Z E^{2} .
\end{array}
$$

The comparison of experimental data and parametrization results shown in Fig. 1 for deuterons incident on nuclei from ${ }^{27} \mathrm{Al}$ to ${ }^{232} \mathrm{Th}$, at energies up to $80 \mathrm{MeV}$, has proved a suitable 


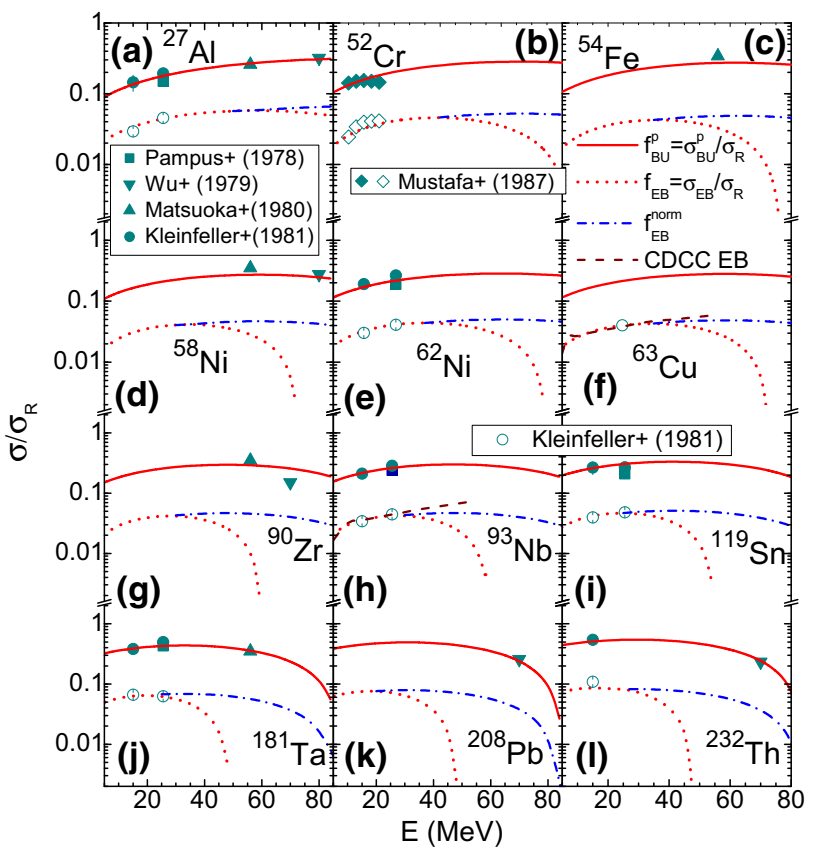

Fig. 1 Comparison of experimental [14,48-50] total breakup protonemission (solid symbols) and elastic-breakup (open circles) fractions of the deuteron total-reaction cross section, and the corresponding parametrization [47] (solid and dotted curves, respectively) as well as the normalized EB fractions [55] (dash-dotted), for deuterons incident on nuclei from ${ }^{27} \mathrm{Al}$ (a) to ${ }^{232} \mathrm{Th}$ (1) at energies up to $80 \mathrm{MeV}$, and $\mathrm{CDCC}$ results for $\mathrm{EB}$ on ${ }^{63} \mathrm{Cu}$ and ${ }^{93} \mathrm{Nb}$ [54] (dashed)

agreement [55]. On the other hand, it was found an apparent decrease of the fraction $f_{E B}$ at energies beyond the range $E<30 \mathrm{MeV}$ of the EB data [14,16], unlike the trend of both the fraction $f_{B U}^{p}$ and the total-reaction cross section.

Because of that, the correctness of the extrapolation of EB parametrization has been checked [54] by comparison with results of the microscopic CDCC method [35]. Thus, a normalization factor has been introduced [55] for extrapolation of $f_{E B}$ at energies beyond the available data, in agreement with the behavior of $f_{B U}^{p}$ [47] and the CDCC calculation results [54]. Hence, we have chosen to keep unchanged the ratio of the $\mathrm{EB}$ and $\mathrm{BU}$ fractions at incident energies above an energy $E_{\max }$ of the $f_{E B}$ maximum [55], by means of the relation:

$$
f_{E B}^{n o r m}(E)=f_{B U}^{n / p}(E) \frac{f_{E B}\left(E_{\max }\right)}{f_{B U}^{n / p}\left(E_{\max }\right)}, \quad E>E_{\max }
$$

Therefore, the normalized EB fraction (dotted curves) follows the behavior of the total $\mathrm{BU}$ nucleon-emission fraction shown in Fig. 1 to be in agreement with the CDCC results [54]. Despite the EB component is less than $10 \%$ of total BU cross section, this $f_{E B}$ normalization is of particular interest at deuteron energies above $\sim 50 \mathrm{MeV}$ and especially for heavier target nuclei, for the inelastic breakup fraction

$f_{B F}^{n / p}=f_{B U}^{n / p}-f_{E B}^{n o r m}$,

as well as the total breakup fraction

$f_{B U}=2 f_{B U}^{n / p}-f_{E B}^{n o r m}$,

under the above-mentioned assumption of equal neutron- and proton-emission BU cross sections $[16,47,55]$. On the other hand, since the corresponding data errors amount to $10-15 \%$ [47], a similar accuracy has been considered also for the present parametrization.

\subsection{Additional BU constraint for $A>200$}

The total BU proton-emission data systematics for heaviest nuclei $(A>200)$ at incident energies around the Coulomb barrier, of great interest for deuteron interaction with actinides nuclei, includes only one single point for ${ }^{232} \mathrm{Th}$ at $E=15$ $\mathrm{MeV}$ [14]. The parametrization [55] describes it properly as well as the value at the incident energy of $70 \mathrm{MeV}$ reported by Wu et al. [49] (Fig. 1).

However, following the EB fraction normalization by Eqs. (3) and (5), the total BU fraction corresponding to this target nucleus may exceed unity. To avoid this unphysical overrun of the total-reaction cross section, and taking into account the above-mentioned systematics accuracy, an additional constraint was adopted for $A>200$, namely 0.9 for the $f_{B U}$ maximum value. Nevertheless, the empirical parametrization should be confirmed by further data measurements and also advanced theoretical modeling.

\subsection{Additive empirical parametrization and microscopic studies}

The recent detailed analyzes of deuteron both $\mathrm{EB}$ and $\mathrm{BF}$ components by the DWBA method [76,77] performed a successful description of proton spectra and angular distributions for the $(d, p)$ reaction on ${ }^{27} \mathrm{Al},{ }^{58} \mathrm{Ni},{ }^{93} \mathrm{Nb}$, and ${ }^{118} \mathrm{Sn}$ at incident energies from $15 \mathrm{MeV}$ to $100 \mathrm{MeV}$. The corresponding calculated EB and BF cross sections would be, however, also quite useful for the comparison with experimental data [14,48-50]. More recently, Neoh et al. [76] applied a CDCC extension, using also microscopic optical potentials, to the analysis of the EB and neutron removal cross sections at 28 $\mathrm{MeV} /$ nucleon on various target nuclei from ${ }^{12} \mathrm{C}$ to ${ }^{209} \mathrm{Bi}$, of interest for actinides nuclei [57,78-80] and further studies of unstable-nuclei structure.

Consequently, the measured total $\mathrm{BU}$ proton-emission cross sections $\sigma_{B U}^{p}$ at $15,25.5,56,70$ and $80 \mathrm{MeV}$ deuteron energies and for target nuclei from ${ }^{12} \mathrm{C}$ to ${ }^{232} \mathrm{Th}[14,48-50]$ are compared in Fig. 2 with the above-described parametrization and results of CDCC extension [76] and DWBA method [77]. Because the absolute cross sections may depend 


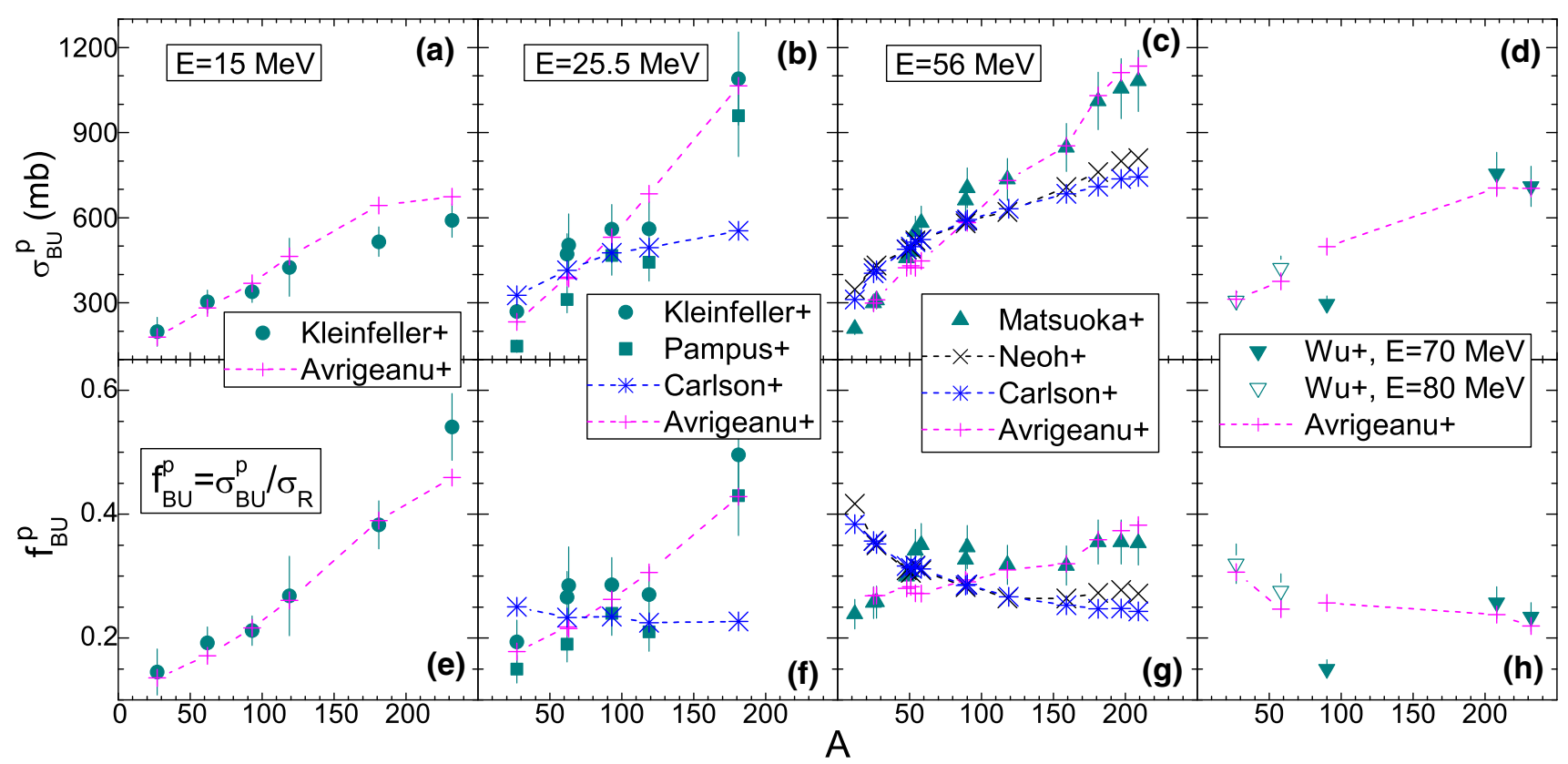

Fig. 2 Comparison of the mass dependence of measured [14,48-50] deuteron total BU proton-emission (top) cross sections and (bottom) fractions, with predictions of the microscopic model [76] (×), DWBA formalism [77] (*), and empirical parametrization $(+)$, connected by dashed lines for eye guiding, for target nuclei from ${ }^{12} \mathrm{C}$ up to ${ }^{209} \mathrm{Bi}$, at deuteron incident energies of $15,25.5,56,70$, and $80 \mathrm{MeV}$ on reaction-model ingredients involved within data analysis, as OMP and PE parameters, a similar comparative analysis concerns at the same time in Fig. 2e-h the corresponding fractions $f_{B U}^{p}$. On the other hand, the $f_{B U}^{p}$ values may illustrate the importance of the breakup process among the other reaction mechanisms related to the deuteron interactions, while the same scale has been used at all incident energies to make also possible an assessment of the data energy dependence.

Thus it results that increase of $\sigma_{B U}^{p}$ with the target-nucleus mass is well described by the empirical parametrization for all deuteron energies from 15 to $80 \mathrm{MeV}$. A similar trend of the microscopic results for medium-mass nuclei with $40<A<120$, but an overestimation of the measured data are apparent for light nuclei $(A<40)$ at both 25 and $56 \mathrm{MeV}$ incident energies, as well as an underestimation for heavier ones $(A>120)$. Moreover, the importance of the BU mechanism, shown by $f_{B U}^{p}$, is increasing with the target-nucleus mass, from ${ }^{27} \mathrm{Al}$ up to ${ }^{232} \mathrm{Th}$, at the lower incident energies of 15 and $25.5 \mathrm{MeV}$. This increase is less significant at the energy of $56 \mathrm{MeV}$, and even reversed at $70-80 \mathrm{MeV}$ so that it seems that the fraction $f_{B U}^{p}$ has reached its maximum at $56 \mathrm{MeV}$ for the target nuclei with $A>120$. Then, this maximum moves at energies over $56 \mathrm{MeV}$ for $40<A<120$, and is still increasing with the incident energy even at $80 \mathrm{MeV}$ for light target nuclei $(A<40)$. The energy dependence of the measured $f_{B U}^{p}$ are satisfactorily described by the empirical parametrization, while the microscopic results are almost constant for all nuclei at 25 $\mathrm{MeV}$ [77], show a steep decrease from $A=12$ up to $A \sim 120$ at $56 \mathrm{MeV}[76,77]$, apart from the data, and underestimate but describe however the $A$-dependence for $A>120$.

Nevertheless, the recently increased interest on the theoretical analysis of the breakup components, e.g. Refs. [8185], may lead eventually to improvement of technologically relevant deuteron-breakup empirical parametrization, already involved within successful analysis of all available data for various nuclei $[18,53,56,58,60-65]$, as well as additional validation and progress of microscopic calculations. It is noticeable in this respect the challenging task found by Potel et al. [84] for the future of the latter topic to be discrimination of the different non-elastic breakup contributions at least in energy regions where they are competing.

\subsection{Deuteron inelastic-breakup enhancement}

While the former effect of BU consideration should be the decrease of $\sigma_{R}$ that is shared among different outgoing channels, by the $\sigma_{B U}$ value, the latter one concerns the BF contributions to different reaction channels. Thus, the absorbed proton or neutron following the deuteron breakup contributes to enhancement of the corresponding $(d, x n)$ or $(d, x p)$ reaction cross sections, respectively. The compound nuclei in reactions induced by BF nucleons differ by one unit of the atomic mass and maybe of also the atomic number than that one in deuteron-induced reactions on the same target nucleus. Moreover, the partition of the BF cross section among various residual-nuclei population is triggered by the energy 
spectra of the BF nucleons and the excitation functions of the reactions induced by these nucleons on the same target nucleus.

Hence, in order to calculate the BF enhancement of, e.g., the $(d, x n)$ reaction cross sections, the BF proton-emission cross section $\sigma_{B F}^{p}$ should be (i) multiplied by the protonenhancing ratios $\sigma_{(p, x)} / \sigma_{R}^{p}$, (ii) convoluted with the Gaussian line shape distribution of the BF-proton energy $E_{p}$ for a given deuteron incident energy $E$ [56-58], and (iii) integrated over the BF-proton energy. Consequently, the BFenhancement cross section has the form [59-61,64]:

$$
\begin{aligned}
\sigma_{B F}^{p, x}(E)= & \sigma_{B F}^{p}(E) \int d E_{p} \frac{\sigma_{(p, x)}\left(E_{p}\right)}{\sigma_{R}^{p}} \\
& \frac{1}{(2 \pi)^{\frac{1}{2}} w} \exp \left[-\frac{\left(E_{p}-E_{p}^{0}(E)\right)^{2}}{2 w^{2}}\right],
\end{aligned}
$$

where $\sigma_{R}^{p}$ is the proton total-reaction cross section, $x$ stands for various $\gamma, n, d$, or $\alpha$ outgoing channels, while the Gaussian distribution parameters $w$ and $E_{p}^{0}$ given by Kalbach [51] were used.

Similarly, the BF enhancement of the $(d, x p)$ reaction cross sections brought by breakup neutrons is given by the following expression:

$$
\begin{aligned}
\sigma_{B F}^{n, x}(E)= & \sigma_{B F}^{n}(E) \int d E_{n} \frac{\sigma_{(n, x)}\left(E_{n}\right)}{\sigma_{T}^{n}} \\
& \frac{1}{(2 \pi)^{\frac{1}{2}} w} \exp \left[-\frac{\left(E_{n}-E_{n}^{0}(E)\right)^{2}}{2 w^{2}}\right],
\end{aligned}
$$

where $\sigma_{T}^{n}$ is the neutron total-reaction cross section, and $\sigma_{(n, x)} / \sigma_{T}^{n}$ are the neutron-enhancing ratios.

Interpolation of experimental nucleon-induced reaction cross sections from the EXFOR library [86] or from newest TENDL library has been involved within estimation of the BU enhancement [53,56-58,60-65,80,87], in order to reduce as much as possible the supplementary uncertainties brought by additional theoretical calculations. In the present work the evaluated neutrons and protons cross sections have been taken from the TENDL-2019 library [9].

On the whole, the enhancing effect of the breakup mechanism is important mainly for describing the excitation functions of second and third chance emitted-particle channels [53,56-58,60-65, 80,87]. This is shown in Fig. 3 for the BF enhancements brought by the breakup-nucleon interactions with various target nuclei from ${ }^{55} \mathrm{Mn}$ up to ${ }^{231} \mathrm{~Pa}$. Particular comments may concern the BF contribution that is even larger than $\mathrm{PE}+\mathrm{CN}$ ones for the $(d, 2 p)$ reaction at all incident energies (Fig. 3d) as well as for $(d, 2 n)$ reaction above $30 \mathrm{MeV}$ (Fig. 3b) and ( $d, 3 n)$ reaction just above the effective threshold (Fig. 3c). Moreover, the entire excitation functions of also $(d, n \alpha),(d, p \alpha)$, and $(d, t)$ would be not described without $\mathrm{BF}$ consideration despite of the dominant nature of the corresponding PE $+\mathrm{CN}$ components shown in Fig. 3e-h.

\section{Discussion}

Selection of the breakup formalism in TALYS code is available by means of the breakupmodel keyword, with value 1 for Kalbach's [19] and 2 for Avrigeanu et al. models. The latter uses Avrigeanu et al. parametrization [12,47,54,55] to calculate the total nucleon-emission, elastic, and inelastic breakup fractions by Eqs. 1, 2, 4, respectively, the corresponding cross sections $\sigma_{B U / B F}^{n / p}=f_{B U / B F}^{n / p} * \sigma_{R}$ and $\sigma_{E B}=$ $f_{E B} * \sigma_{R}$, as well as the BU neutron- and proton-emission spectra on the basis of the former Gaussian parametrization of Kalbach [51]. A distinct note should concern the maximum energy of the BU nucleons spectra which is the difference between the deuteron incident energy and its binding energy.

Verification and validation (V\&V) [7] of the advanced breakup account in addition to TALYS-1.95 has been carried out for deuteron-induced reactions on ${ }^{58} \mathrm{Ni},{ }^{96} \mathrm{Zr}$, and ${ }^{231} \mathrm{~Pa}$ target nuclei, up to $200 \mathrm{MeV}$ incident energy. Of particular interest for the present work is the comparative analysis of the results of TALYS-1.95 using both the option breakupmodel $\mathbf{1}$, and option breakupmodel $\mathbf{2}$ with the additional breakup enhancement detailed within present work.

First, an outline of the additional breakup enhancement is made possible by comparison of the TALYS-1.95 default output and results of the implicit BU-enhancement addition in the cases of activation excitation functions of residual nuclei ${ }^{64} \mathrm{Cu}$ and ${ }^{96} \mathrm{Nb}$ by $(d, x n)$ reactions on natural $\mathrm{Ni}$ and $\mathrm{Zr}$, respectively (Fig. 4a,c). The latter values are increased by $57 \%$ and $86 \%$, respectively, with respect to the former ones at a reference deuteron energy of $40 \mathrm{MeV}$. Nevertheless, results identical with the TALYS-1.95 default output are obtained by using the option breakupmodel 1 also within latest code version, which includes the BU enhancement only for the breakupmodel 2 option.

The BF explicit contribution by means of the $(p, n)$ reactions induced by the BU-protons on the same target nuclei may be shown for distinct isotopes, e.g. the heaviest stable ones, in Fig. 4b,d. Actually they were selected due to the corresponding larger $(x, 2 n)$ reaction cross sections. These BF contributions amount to $\sim 36 \%$ and $46 \%$, respectively, of the total activation cross sections of the two residual nuclei at the same reference deuteron energy of $40 \mathrm{MeV}$.

Second, a comparative analysis of the total-BU and BU nucleon-emission excitation functions corresponding to Kalbach [51] and Avrigeanu et al. [55] parametrizations is shown in Fig. 5a,e,i. While Kalbach's BU excitation functions decrease slowly from their maxima to $200 \mathrm{MeV}$, our approach led to a strong energy dependence and vanishing 


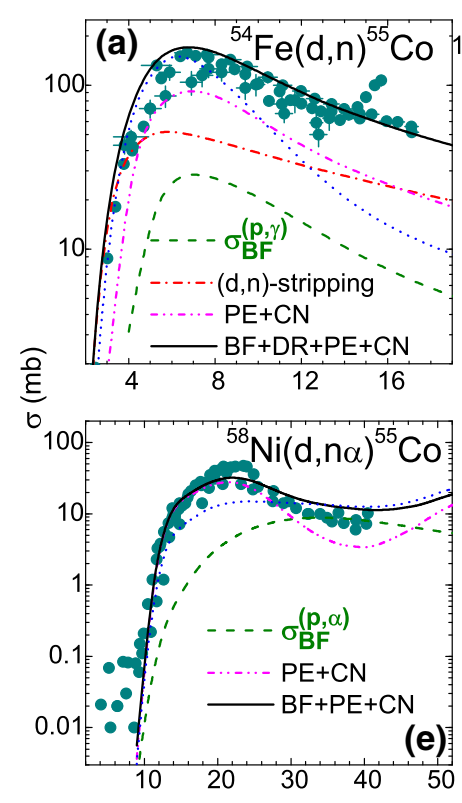

(e)
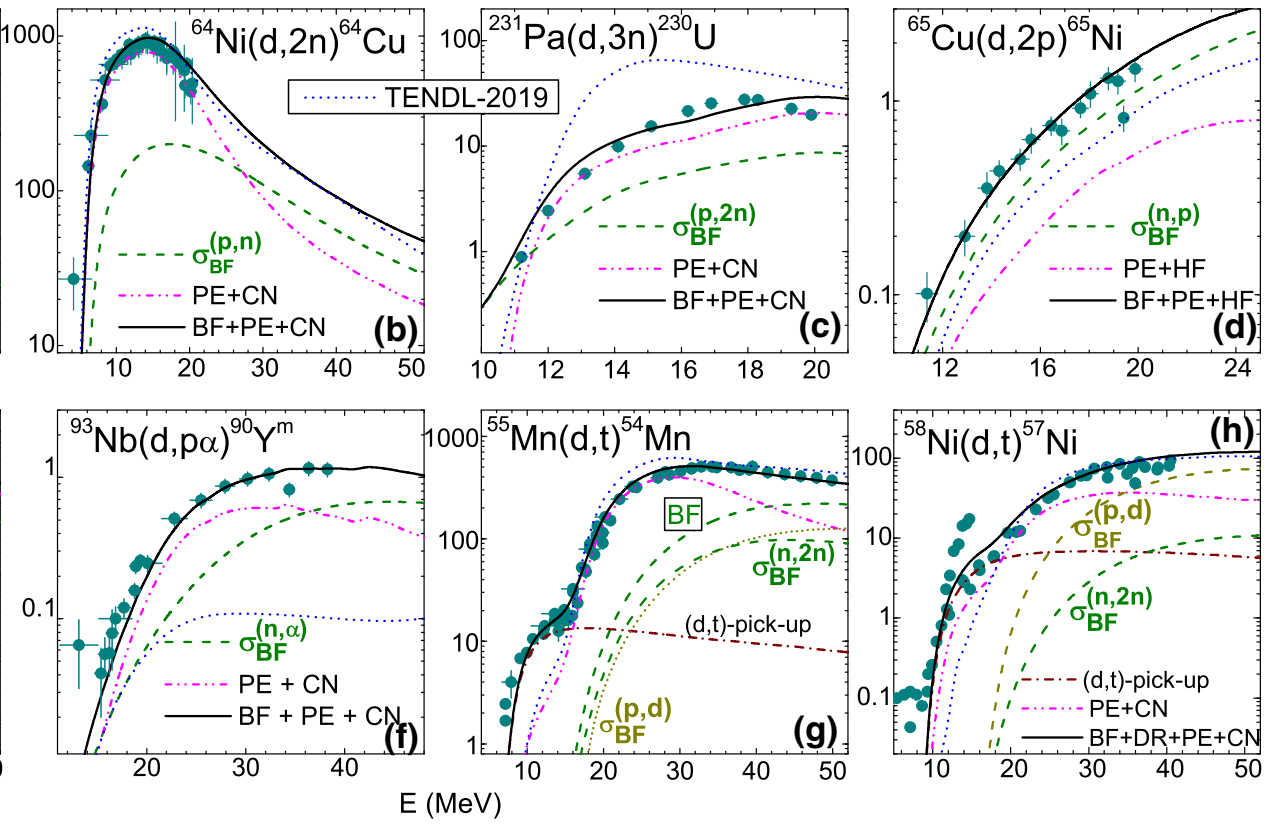

Fig. 3 Comparison of deuteron-activation cross sections measured [86], TENDL-2019 evaluation (dotted curves), and calculated results (thick solid curves) $[56-58,60,61,64]$ taking into account the BF

enhancement (dashed curves), direct reactions, stripping/pick-up, (dash-dotted curves), pre-equilibrium (PE), and compound nucleus (CN) contributions (dash-dot-dotted curves)

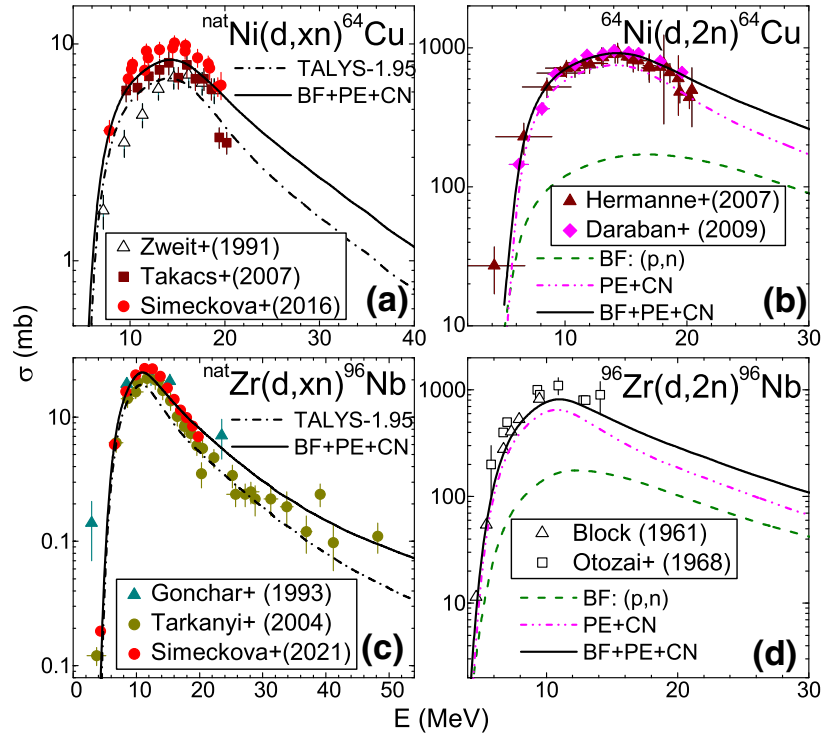

Fig. 4 Comparison of measured [86] and calculated cross sections using the code TALYS-1.95 with default options (dash-dotted curves) as well as latter breakup-model option and including the additional breakup enhancement (solid curves) for deuteron-induced activation of ${ }^{64} \mathrm{Cu}$ and ${ }^{96} \mathrm{Nb}$ a, $\mathbf{c}$ on natural $\mathrm{Ni}$ and $\mathrm{Zr}$, respectively, and $\mathbf{b}, \mathbf{d}$ heaviest stable isotopes ${ }^{64} \mathrm{Ni}$ and ${ }^{96} \mathrm{Zr}$, with $\mathrm{BF}$ (dashed cureves) and $\mathrm{PE}+\mathrm{CN}$ (dash-dot-dotted) contributions also shown

around $100 \mathrm{MeV}$, faster for heavier target nuclei. Obviously, such differences in BU excitation functions have corresponding effects on the activation excitation functions shown in Fig. $5 b-d, f-h, j-1)$.
The comparative analysis of these functions reveals both complementary effects of the breakup mechanism: overall decreasing of the excitation functions due the leakage of initial deuteron flux to the breakup process, and the inelastic breakup enhancement. Due to the restrictive energy range of the experimental BU fractions [14,48-50] involved for settling our parametrization [55], the corresponding excitation functions for BF enhancement shown in Fig. 5 are not going over $80 \mathrm{MeV}$. Nevertheless, the $80 \mathrm{MeV}$ deuteron incidentenergy limit is still appropriate for the EUROfusion objectives, while an eventual switch to the default TALYS-1.95 results seems practical at higher energies.

On the other hand, the default TALYS-1.95 results and the statistical $\mathrm{PE}+\mathrm{CN}$ contributions corresponding to latter breakup-model option, including the additional breakup enhancement, are rather close (dash-dotted and dash-dotdotted curves, respectively, in Fig. 5). The effects of breakup enhancement, that are also is evidenced by the difference between the total excitation functions and the $\mathrm{PE}+\mathrm{CN}$ contributions, are larger for the $(d, 2 n)$ reactions and lighter target nuclei. However, the breakup enhancement becomes significant also for the heavier nuclei at deuteron energies above $\sim$ $40 \mathrm{MeV}$. 


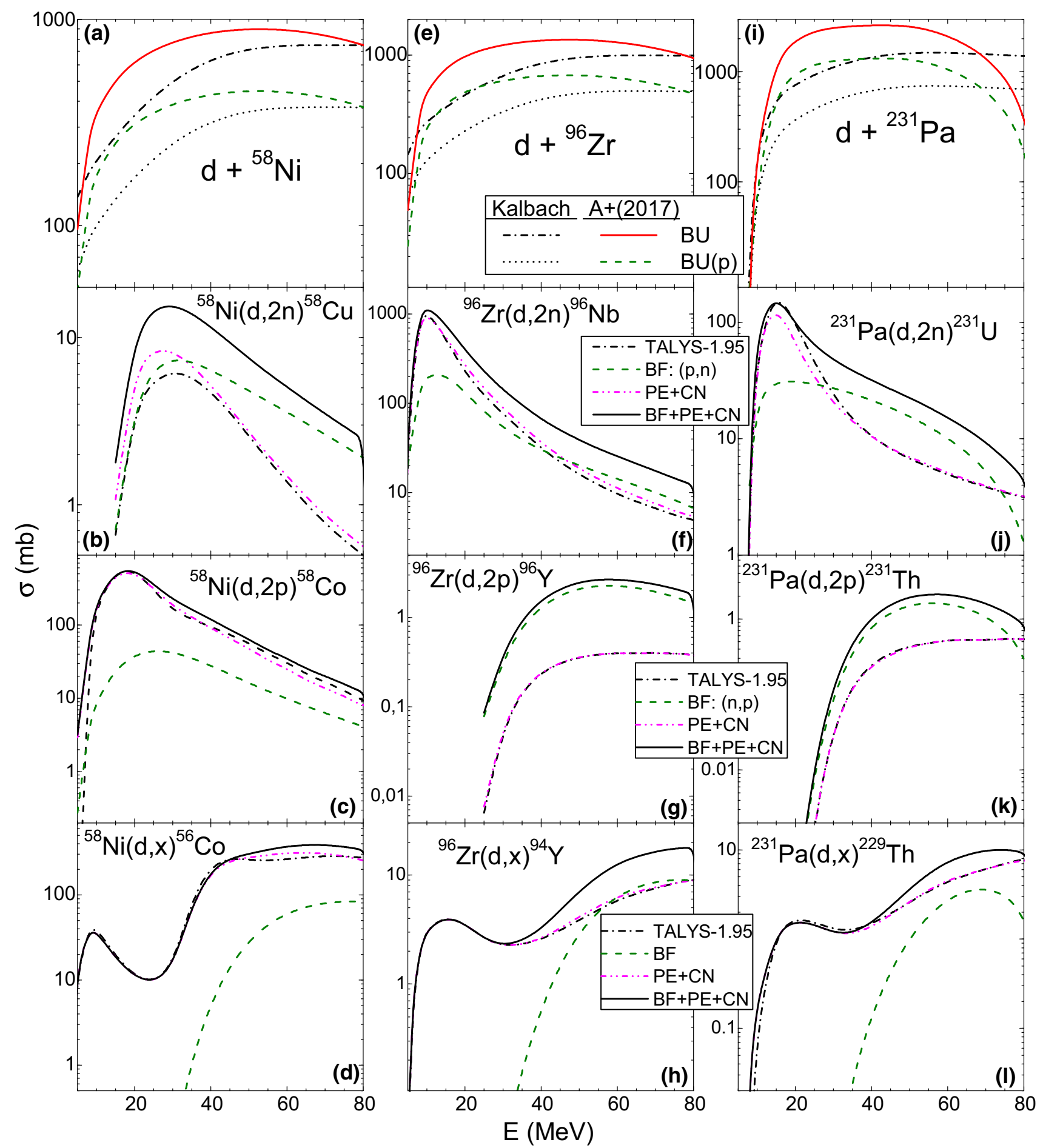

Fig. 5 Comparison of calculated excitation functions of the code TALYS-1.95 with default options (dash-dotted curves) as well as latter breakup-model option and including the additional breakup enhancement (solid curves) for: a, e, i total BU and also BU proton-emission (dashed and dotted curves, respectively) for deuterons incident on ${ }^{58} \mathrm{Ni}$, ${ }^{96} \mathrm{Zr}$, and ${ }^{231} \mathrm{~Pa}$, and $\mathbf{b}-\mathbf{d}, \mathbf{f}-\mathbf{h}, \mathbf{j}-\mathbf{l}$ activation of residual nuclei for the same target nuclei, including also BF (dashed curves) and PE + CN (dash-dot-dotted) contributions 


\section{Summary and conclusions}

Subsequently to the EUROfusion PPPT-programme action for an advanced modeling approach of deuteron reaction cross sections [11], as well as specific data evaluations [53,56-58,60-65] in addition of the TENDL files, an assessment of the details and corresponding outcome related to a latter option of TALYS for the breakup model $[12,47,55]$ has been carried out. It is based on an empirical parametrization of both the total breakup (EB + BF) and EB data, under the assumption that the inelastic-breakup cross section for neutron emission $\sigma_{B F}^{n}$ is the same as that for the proton emission $\sigma_{B F}^{p}$ [16]. Actually, the parametrization has concerned the total BU nucleon-emission as well as EB fractions, i.e. $f_{B U}^{n / p}$ $=\sigma_{B U}^{n / p} / \sigma_{R}$ and $f_{E B}=\sigma_{E B} / \sigma_{R}$, respectively. The dependence of these fractions on the deuteron incident energy $E$ and the target-nucleus atomic $Z$ and mass $A$ numbers was obtained $[47,55]$ through analysis of the experimental systematics of deuteron-induced reactions on target nuclei from ${ }^{27} \mathrm{Al}$ to ${ }^{232} \mathrm{Th}$ and incident energies up to $80 \mathrm{MeV}$ for the former [14,48-50]. Moreover, a normalization factor has been introduced [55] for extrapolation of $f_{E B}$ at energies beyond the available data, in agreement with the behavior of $f_{B U}^{p}$ [47] and the CDCC calculation results [54]. At the same time, an additional constraint was adopted for $A>200$, namely 0.9 for the $f_{B U}$ maximum value.

On the other hand, while the $\sigma_{R}$ value is decreased by the total-breakup cross section, the nucleons following the deuteron breakup and absorbed by the same target nucleus contributes to the enhancement of the $(d, x)$ reaction cross sections through corresponding $(n, x)$ and $(p, x)$ reactions. The partition of the BF cross section among various residualnuclei population is triggered by the energy spectra of the $\mathrm{BF}$ nucleons and the excitation functions of the reactions induced by these nucleons on the same target nucleus. Consequently, in order to calculate the BF enhancement of, e.g., the $(d, x n)$ reaction cross sections, the $\mathrm{BF}$ proton-emission cross section $\sigma_{B F}^{p}$ should be (i) multiplied by the proton-enhancing ratios $\sigma_{(p, x)} / \sigma_{R}^{p}$, with the evaluated proton-induced reaction data of TENDL-2019 concerned, (ii) convoluted with the Gaussian line shape distribution [51] of the BF-proton energy $E_{p}$ for a given deuteron incident energy $E$ [56-58], and (iii) integrated over the BF-proton energy. Actually, the former breakupenhancement account [53] corresponds to the 2nd and 3rd statistical-decay components [70] within former DEURACS framework, weighted by nonelastic-breakup formation fractions $R_{n}$ and $R_{p}$ calculated with the Glauber model at half of the incident-deuteron energy. At the same time, the final one $[59,60]$ corresponds to the DEURACS use of energydependent fractions [44] $R_{n}\left(E_{n}\right)$ and $R_{p}\left(E_{p}\right)$ also within Glauber model.
On the whole, the enhancing effect of the breakup mechanism is important mainly for describing the excitation functions for second and third chance emitted-particle channels [53,56-58,60-65,80,87]. Particular comments concerned the $\mathrm{BF}$ contribution even larger than the $\mathrm{PE}+\mathrm{CN}$ ones for the $(d, 2 p)$ reaction for heavier nuclei at all incident energies, as well as $(d, 2 n)$ reaction above $30 \mathrm{MeV}$, and even $(d, 3 n)$ reaction just above the effective threshold (Fig. 5). Moreover, the entire excitation functions of also $(d, n \alpha),(d, p \alpha)$, and $(d, t)$ would be not described without BF consideration despite of the dominant nature of the corresponding $\mathrm{PE}+\mathrm{CN}$ components (Fig. 3).

Verification and validation of the advanced breakup account in addition to TALYS-1.95 has been carried out for deuteron-induced reactions on ${ }^{58} \mathrm{Ni},{ }^{96} \mathrm{Zr}$, and ${ }^{231} \mathrm{~Pa}$ target nuclei. Thus it has been found that the BU-enhancement addition increases by $57 \%$ and $86 \%$ the activation cross sections of residual nuclei ${ }^{64} \mathrm{Cu}$ and ${ }^{96} \mathrm{Nb}$ in $(d, x n)$ reactions on natural $\mathrm{Ni}$ and $\mathrm{Zr}$, respectively, at a reference deuteron energy of $40 \mathrm{MeV}$. The BF contribution through $(p, n)$ reaction induced by the BU-protons on the heaviest stable isotopes ${ }^{64} \mathrm{Ni}$ and ${ }^{96} \mathrm{Zr}$, corresponding to larger $(d, 2 n)$ reaction cross sections, amounts to $\sim 36 \%$ and $46 \%$, respectively, of their total activation cross sections at the same reference deuteron energy of $40 \mathrm{MeV}$. Moreover, the effects of breakup enhancement are larger for the $(d, 2 n)$ reactions and lighter target nuclei. However, they become significant also for the heavier nuclei at deuteron energies above $\sim 40 \mathrm{MeV}$.

Actually, the same approach beyond elastic-scattering analysis for OMP validation, using BU parametrization [47,55] for the related deuteron $\sigma_{R}$ decrease as well as breakup-enhancement of various $(d, x)$ reaction cross sections, DWBA spectroscopic factors and related uncertainties [65] from data analysis with FRESCO of available particleemission angular distributions, and $\mathrm{PE}+\mathrm{CN}$ framework of either STAPRE-H or then TALYS codes has been consistently involved within suitable account of all available excitation functions of deuterons up to $60 \mathrm{MeV}$ on ${ }^{27} \mathrm{Al}$ [53], ${ }^{63,65, \text { nat }} \mathrm{Cu}[18,56],{ }^{93} \mathrm{Nb}[58],{ }^{54,56,57,58, \text { nat }} \mathrm{Fe}$ [60],

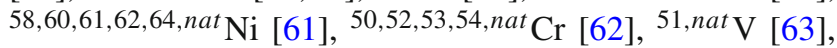
${ }^{59} \mathrm{Co}$ [63], ${ }^{55} \mathrm{Mn}$ [64], and ${ }^{90,91,92,94,96, \text { nat }} \mathrm{Zr}$ [65]. Due consideration of all $\mathrm{EB}, \mathrm{BF}, \mathrm{DR}, \mathrm{PE}$, and $\mathrm{CN}$ reaction mechanisms $[60,61]$ is indeed found crucial [66] for a consistent analysis of the deuteron-reaction measured data and even high production of proton-rich nuclei [67], while insufficient treatment and separation between different reaction mechanisms such as DR and BU components [68] may be related to deviations between measurements and advanced surrogate reaction studies [69].

Nevertheless, the updated approach detailed in this work can be used to provide more accurate deuteron-reaction data that are also eventually required during the deuteron crosssection V\&V activities within EUROfusion/ENS, with addi- 
tion of only the particular $(d, p)$ and $(d, t)$ reaction contribution to be obtained by using the direct-reaction DWBA method as also it is performed within the code system DEURACS for deuterons incident on light as well as increasingmass nuclei.

Acknowledgements This work has been partly supported by Autoritatea Nationala pentru Cercetare Stiintifica (Project PN-19060102) and carried out within the framework of the EUROfusion Consortium and has received funding from the Euratom research and training programme 2014-2018 and 2019-2020 under Grant agreement No 633053. The views and opinions expressed herein do not necessarily reflect those of the European Commission.

Data Availability Statement This manuscript has no associated data or the data will not be deposited. [Authors' comment: This is a model analysis of the published data, and results of all calculations are displayed in the figures and can be reproduced by using the parameter values given or properly referenced. Additional data related to this work are available from the authors on request.]

Open Access This article is licensed under a Creative Commons Attribution 4.0 International License, which permits use, sharing, adaptation, distribution and reproduction in any medium or format, as long as you give appropriate credit to the original author(s) and the source, provide a link to the Creative Commons licence, and indicate if changes were made. The images or other third party material in this article are included in the article's Creative Commons licence, unless indicated otherwise in a credit line to the material. If material is not included in the article's Creative Commons licence and your intended use is not permitted by statutory regulation or exceeds the permitted use, you will need to obtain permission directly from the copyright holder. To view a copy of this licence, visit http://creativecomm ons.org/licenses/by/4.0/.

\section{References}

1. A. Moeslang, V. Heinzel, H. Matsui, M. Sugimoto, Fusion Eng. Des. 81, 863 (2006)

2. ITER Organization, http://www.iter.org/. Accessed 29 Oct 2021

3. G. Federici, C. Bachmann, W. Biel, L. Boccaccini, F. Cismondi, S. Ciattaglia, M. Coleman, C. Day, E. Diegele, T. Franke et al., Fusion Eng. Des. 109-111, 1464 (2016). (proceedings of the 12th International Symposium on Fusion Nuclear Technology-12 (ISFNT-12))

4. EUROfusion programme, https://users.euro-fusion.org/. Accessed 29 Oct 2021

5. Fusion for Energy, https://fusionforenergy.europa.eu/. Accessed 29 Oct 2021

6. U. Fischer, M. Avrigeanu, V. Avrigeanu, O. Cabellos, N. Dzysiuk, A. Koning, A. Klix, A. Konobeev, I. Kodeli, H. Leeb et al., EPJ Web Conf. 146, 09003 (2017)

7. D. Leichtle, Considerations on V\&V needs for FENDL data and application, in FENDL Library for Fusion Neutronics Calculations. Summary report from the Technical Meeting, 2-5 Sept. 2019 , Vienna, Austria, Report INDC(NDS)-0797, ed. by D. Leichtle, A. Trkov (IAEA, Vienna, 2019), p. 15

8. R.A. Forrest, R. Capote, N. Otsuka, T. Kawano, A.J. Koning, S. Kunieda, J.C. Sublet, Y. Watanabe, Tech. rep., IAEA, Vienna (2012). https://www-nds.iaea.org/publications/ indc/indc-nds-0628.pdf. Accessed 29 Oct 2021
9. A. Koning, D. Rochman, J.C. Sublet, N. Dzysiuk, M. Fleming, S. van der Marck, Nucl. Data Sheets 155, 1 (2019). (special Issue on Nuclear Reaction Data)

10. A.J. Koning, S. Hilaire, M.C. Duijvestijn, TALYS-1.0, in Proceedings of the International Conference on Nuclear Data for Science and Technology - ND2007, April 22-27, 2007, Nice, France, ed. by O. Bersillon, F. Gunsing, E. Bauge, R. Jacqmin, S. Leray (EDP Sciences, 2008), pp. 211-214. http://www.talys.eu. Accessed 29 Oct 2021

11. U. Fischer, M. Avrigeanu, V. Avrigeanu, A. Konobeyev, I. Kodeli, H. Leeb, M. Fleming, M. Gilbert, D. Rochman, P. Pereslavtsev et al., EPJ Web Conf. 239, 21001 (2020)

12. M. Avrigeanu, V. Avrigeanu, Phys. Rev. C 92, 021601 (2015)

13. U. Fischer, M. Angelone, M. Avrigeanu, V. Avrigeanu, C. Bachmann, N. Dzysiuk, M. Fleming, A. Konobeev, I. Kodeli, A. Koning et al., Fusion Eng. Des. 136, 162 (2018). (special Issue: Proceedings of the 13th International Symposium on Fusion Nuclear Technology (ISFNT-13))

14. J. Kleinfeller, J. Bisplinghoff, J. Ernst, T. Mayer-Kuckuk, G. Baur, B. Hoffmann, R. Shyam, F. Rösel, D. Trautmann, Nucl. Phys. A 370, 205 (1981)

15. G. Baur, F. Rösel, D. Trautmann, R. Shyam, Phys. Rep. 111, 333 (1984)

16. M.G. Mustafa, T. Tamura, T. Udagawa, Phys. Rev. C 35, 2077 (1987)

17. X. Ledoux, M. Aïche, M. Avrigeanu, V. Avrigeanu, E. Balanzat, B. Ban-d'Etat, G. Ban, E. Bauge, G. Bélier, P. Bém et al., Radiat. Prot. Dosim. 180, 115 (2017)

18. I. Mardor, O. Aviv, M. Avrigeanu, D. Berkovits, A. Dahan, T. Dickel, I. Eliyahu, M. Gai, I. Gavish-Segev, S. Halfon et al., Eur. Phys. J. A 54, 91 (2018)

19. C. Kalbach, Phys. Rev. C 95, 014606 (2017)

20. C. Kalbach, Phys. Rev. C 71, 034606 (2005)

21. Charged-particle cross section database for medical radioisotope production and beam monitor reactions, https://www-nds.iaea.org/ medical/. Accessed 29 Oct 2021

22. A. Ignatyuk, Test of the FENDL-3.1 activation data against the revised experimental data for the charged-particle induced reactions, in FENDL Library for Fusion Neutronics Calculations. Summary report from the Technical Meeting, 2-5 Sept. 2019, Vienna, Austria, Report INDC(NDS)-0797, ed. by D. Leichtle, A. Trkov (IAEA, Vienna, 2019), p. 10

23. A. Hermanne, A.V. Ignatyuk, R. Capote, B.V. Carlson, J.W. Engle, M.A. Kellett, T. Kibédi, G. Kim, F.G. Kondev, M. Hussain et al., Nucl. Data Sheets 148, 338 (2018). (special Issue on Nuclear Reaction Data)

24. J. Engle, A. Ignatyuk, R. Capote, B. Carlson, A. Hermanne, M. Kellett, T. Kibédi, G. Kim, F. Kondev, M. Hussain et al., Nucl. Data Sheets 155, 56 (2019). (special Issue on Nuclear Reaction Data)

25. A.V. Ignatyuk, Evaluations of (d,p) cross sections, in FENDL3 Library Final Report of the Coordinated Research Project on Nuclear Data Libraries for Advanced Systems: Fusion Devices, Dec. 2013, Vienna, Austria, Report INDC(NDS)-0645, ed. by R.A. Forrest, C.W. Kalbach, M. Avrigeanu, V. Avrigeanu, A.V. Ignatyuk, F. Tárkányi, A. Trkov, J. Kopecky, U. Fischer (IAEA, Vienna, 2013), p. 56

26. F. Tárkányi, A. Hermanne, F. Ditrói, S. Takács, A.V. Ignatyuk, I. Spahn, S. Spellerberg, Eur. Phys. J. A 57, 21 (2021)

27. F. Tárkányi, S. Takács, F. Ditrói, Z. Szúcs, K. Brezovcsik, A. Hermanne, A.V. Ignatyuk, Eur. Phys. J. A 57, 233 (2021)

28. A.J. Koning, D. Rochman, Ann. Nucl. Energy 35, 2024 (2008)

29. M. Avrigeanu, W. von Oertzen, U. Fischer, V. Avrigeanu, Nucl. Phys. A 759, 327 (2005)

30. U. Fischer, M. Avrigeanu, P. Pereslavtsev, S. Simakov, I. Schmuck, J. Nucl. Mater. 367-370, 1531 (2007). (proceedings of the 


\section{Twelfth International Conference on Fusion Reactor Materials} (ICFRM-12))

31. P. Pereslavtsev, U. Fischer, S. Simakov, M. Avrigeanu, Nucl. Instrum. Methods Phys. Res. Sect. B Beam Interact. Mater. Atoms 266, 3501 (2008)

32. I.J. Thompson, Comput. Phys. Rep. 7, 167 (1988). (version FRES 2.9, Sept. 2011 LLNL-SMI)

33. W. von Oertzen, H. Bohlen, Phys. Rep. 19, 1 (1975)

34. T. Ye, Y. Watanabe, K. Ogata, S. Chiba, Phys. Rev. C 78, 024611 (2008)

35. K. Ogata, M. Yahiro, Y. Iseri, T. Matsumoto, M. Kamimura, Phys. Rev. C 68, 064609 (2003)

36. T. Ye, Y. Watanabe, K. Ogata, Phys. Rev. C 80, 014604 (2009)

37. R.J. Glauber, High-Energy Collision Theory, in Lectures in Theoretical Physics (Interscience, New York, 1959), p. 315

38. T. Ye, S. Hashimoto, Y. Watanabe, K. Ogata, M. Yahiro, Phys. Rev. C 84, 054606 (2011)

39. S. Hashimoto, M. Yahiro, K. Ogata, K. Minomo, S. Chiba, Phys. Rev. C 83, 054617 (2011)

40. T. Ye, Y. Watanabe, Nucl. Data Sheets 118, 308 (2014)

41. S. Nakayama, Y. Watanabe, J. Nucl. Sci. Technol. 53, 89 (2016). https://doi.org/10.1080/00223131.2015.1023380

42. S. Nakayama, H. Kouno, Y. Watanabe, O. Iwamoto, T. Ye, K. Ogata, EPJ Web Conf. 122, 04004 (2016)

43. S. Nakayama, H. Kouno, Y. Watanabe, O. Iwamoto, K. Ogata, Phys. Rev. C 94, 014618 (2016)

44. S. Nakayama, K. Hiroshi, Y. Watanabe, O. Iwamoto, T. Ye, K. Ogata, EPJ Web Conf. 146, 12025 (2017)

45. S. Nakayama, O. Iwamoto, Y. Watanabe, K. Ogata, J. Nucl. Sci. Technol. 58, 805 (2021). https://doi.org/10.1080/00223131.2020. 1870010

46. R.A. Forrest, I. Cook, Fusion Eng. Des. 82, 2478 (2007). (proceedings of the 24th Symposium on Fusion Technology)

47. M. Avrigeanu, W. von Oertzen, R.A. Forrest, A.C. Obreja, F.L. Roman, V. Avrigeanu, Fusion Eng. Des. 84, 418 (2009)

48. J. Pampus, J. Bisplinghoff, J. Ernst, T. Mayer-Kuckuk, J. Rama Rao, G. Baur, F. Rösel, D. Trautmann, Nucl. Phys. A 311, 141 (1978)

49. J.R. Wu, C.C. Chang, H.D. Holmgren, Phys. Rev. C 19, 370 (1979)

50. N. Matsuoka, M. Kondo, A. Shimizu, T. Saito, S. Nagamachi, H. Sakaguchi, A. Goto, F. Ohtani, Nucl. Phys. A 345, 1 (1980)

51. C. Kalbach Walker, Tech. rep., IAEA, Vienna (2010), https:// www-nds.iaea.org/fendl3/docs/dBreakupRCM2.pdf. Accessed 29 Oct 2021

52. M. Avrigeanu, V. Avrigeanu, Tech. rep., Institute for Physics and Nuclear Engineering Report NP-86-1995, Bucharest (1995), http:// www.oecd-nea.org/tools/abstract/detail/iaea0971/. Accessed 29 Oct 2021

53. P. Bém, E. Šimečková, M. Honusek, U. Fischer, S.P. Simakov, R.A. Forrest, M. Avrigeanu, A.C. Obreja, F.L. Roman, V. Avrigeanu, Phys. Rev. C 79, 044610 (2009)

54. M. Avrigeanu, A.M. Moro, Phys. Rev. C 82, 037601 (2010)

55. M. Avrigeanu, V. Avrigeanu, Phys. Rev. C 95, 024607 (2017)

56. E. Šimečková, P. Bém, M. Honusek, M. Štefánik, U. Fischer, S.P. Simakov, R.A. Forrest, A.J. Koning, J.C. Sublet, M. Avrigeanu et al., Phys. Rev. C 84, 014605 (2011)

57. M. Avrigeanu, V. Avrigeanu, A.J. Koning, Phys. Rev. C 85, 034603 (2012)

58. M. Avrigeanu, V. Avrigeanu, P. Bém, U. Fischer, M. Honusek, A.J. Koning, J. Mrázek, E. Šimečková, M. Štefánik, L. Závorka, Phys. Rev. C 88, 014612 (2013)

59. M. Avrigeanu, V. Avrigeanu, Nucl. Data Sheets 118, 301 (2014)

60. M. Avrigeanu, V. Avrigeanu, P. Bém, U. Fischer, M. Honusek, K. Katovsky, C. Mănăilescu, J. Mrázek, E. Šimečková, L. Závorka, Phys. Rev. C 89, 044613 (2014)
61. M. Avrigeanu, E. Šimečková, U. Fischer, J. Mrázek, J. Novak, M. Štefánik, C. Costache, V. Avrigeanu, Phys. Rev. C 94, 014606 (2016)

62. E. Šimečková, M. Avrigeanu, U. Fischer, J. Mrázek, J. Novak, M. Štefánik, C. Costache, V. Avrigeanu, Phys. Rev. C 98, 034606 (2018)

63. A. Kreisel, L. Weissman, A. Cohen, T. Hirsh, A. Shor, O. Aviv, I. Eliyahu, M. Avrigeanu, V. Avrigeanu, Phys. Rev. C 99, 034611 (2019)

64. M. Avrigeanu, E. Šimečková, U. Fischer, J. Mrázek, J. Novak, M. Štefánik, C. Costache, V. Avrigeanu, Phys. Rev. C 101, 024605 (2020)

65. E. Šimečková, M. Avrigeanu, J. Mrázek, J. Novak, M. Štefánik, C. Costache, V. Avrigeanu, Phys. Rev. C 104, 044615 (2021)

66. V. Jha, V. Parkar, S. Kailas, Phys. Rep. 845, 1 (2020). (incomplete fusion reactions using strongly and weakly bound projectiles)

67. H. Wang, H. Otsu, N. Chiga, S. Kawase, S. Takeuchi, T. Sumikama, S. Koyama, H. Sakurai, Y. Watanabe, S. Nakayama et al., Commun. Phys. 2, 78 (2019)

68. M. Avrigeanu, V. Avrigeanu, J. Phys. Conf. Ser. 724, 012003 (2016)

69. J.J. Cowan, C. Sneden, J.E. Lawler, A. Aprahamian, M. Wiescher, K. Langanke, G. Martínez-Pinedo, F.K. Thielemann, Rev. Mod. Phys. 93, 015002 (2021)

70. S. Nakayama, S. Araki, Y. Watanabe, O. Iwamoto, T. Ye, K. Ogata, Nucl. Data Sheets 118, 305 (2014)

71. S. Nakayama, S. Araki, Y. Watanabe, O. Iwamoto, T. Ye, K. Ogata, Energy Proc. 71, 219 (2015). (the Fourth International Symposium on Innovative Nuclear Energy Systems, INES-4)

72. Y. Watanabe, T.T. Kin, S.S. Araki, S. Nakayama, O. Iwamoto, EPJ Web Conf. 146, 03006 (2017)

73. C. Kalbach, Zeitschrift für Physik A Atoms and Nuclei 283, 401 (1977)

74. S. Nakayama, N. Furutachi, O. Iwamoto, Y. Watanabe, Phys. Rev. C 98, 044606 (2018)

75. S. Nakayama, O. Iwamoto, Y. Watanabe, Phys. Rev. C 100, 044603 (2019)

76. Y.S. Neoh, K. Yoshida, K. Minomo, K. Ogata, Phys. Rev. C 94, 044619 (2016)

77. B.V. Carlson, R. Capote, M. Sin, Few-Body Syst. 57, 307 (2016)

78. J.N. Wilson, F. Gunsing, L.A. Bernstein, A. Bürger, A. Görgen, M. Guttormsen, A.C. Larsen, P. Mansouri, T. Renstrøm, S.J. Rose et al., Phys. Rev. C 85, 034607 (2012)

79. Q. Ducasse, B. Jurado, M. Aïche, P. Marini, L. Mathieu, A. Görgen, M. Guttormsen, A.C. Larsen, T. Tornyi, J.N. Wilson et al., Phys. Rev. C 94, 024614 (2016)

80. M. Avrigeanu, V. Avrigeanu, Role of the direct mechanisms in the deuteron-induced surrogate reactions, in Proceedings of the 14th International Conference on Nuclear Reaction Mechanisms Varenna, Italy, 2015, ed. by F. Cerutti, M. Chadwick, A. Ferrari, T. Kawano, P. Schoofs (CERN, Geneva, 2015), pp. 203-208

81. G. Potel, F.M. Nunes, I.J. Thompson, Phys. Rev. C 92, 034611 (2015)

82. J. Lei, A.M. Moro, Phys. Rev. C 92, 044616(R) (2015)

83. K. Ogata, K. Yoshida, Phys. Rev. C 94, 051603(R) (2016)

84. G. Potel, G. Perdikakis, B.V. Carlson, M.C. Atkinson, W.H. Dickhoff, J.E. Escher, M.S. Hussein, J. Lei, W. Li, A.O. Macchiavelli et al., Eur. Phys. J. A 53, 178 (2017)

85. J. Lei, A.M. Moro, Phys. Rev. C 97, 011601(R) (2018)

86. Experimental nuclear reaction data (exfor), http://www-nds.iaea. org/exfor/

87. M. Avrigeanu, V. Avrigeanu, Comparative analysis of empirical parametrization and microscopical studies of deuteron-induced reactions, in Proceedings of the 15th International Conference on Nuclear Reaction Mechanisms Varenna, Italy, 2018, ed. by F. Cerutti, A. Ferrari, T. Kawano, P. Salvat-Pujol, P. Talou (CERN, Geneva, 2018), pp. 131-138 